\title{
MODELS OF COMPLETE THEORIES
}

\section{R. L. VAUGHT}

The semantical concepts, such as satisfaction, truth, and model, form the subject matter of a field known as the theory of models. I am going to discuss today several related recent developments in this field. They all lie in one particular area which is indicated by the title and which will be described more fully in a moment. However, some introductory and side remarks I shall make may also serve to indicate to those unfamiliar with the theory of models at least what some of the other areas of the field are.

Perhaps the earliest result in the theory of models, dating from 1915, is the theorem of Löwenheim and Skolem: Any infinite algebraic system has a denumerable subsystem having the same true (elementary) sentences. Before discussing this theorem further, we must define the notions involved in it.

By an algebraic system is meant a system $\mathfrak{A}=\left\langle|\mathfrak{A}|, R_{0}^{\mathfrak{R}}, R_{1}^{\mathfrak{A}}, \ldots\right\rangle$ formed by a nonempty set $|\mathfrak{A}|$ and finitely or denumerably many relations $R_{0}^{\mathfrak{A}}, R_{1}^{\mathfrak{A}}, \ldots$ among the elements of $\mathfrak{A}$, each $R_{n}^{\mathfrak{Q}}$ having a finite number $\rho_{n}$ of places. Thus, for example, an ordered group is a system $\langle G,\langle, \cdot, e\rangle$ having a binary relation, a binary operation (which may be regarded as a special kind of ternary relation), and a distinguished element (a special kind of singulary relation). A system $\mathfrak{B}$, having the same similarity type $\rho$ as $\mathfrak{A}$, is a subsystem of $\mathfrak{A}$ if $|\mathfrak{B}| \subseteq|\mathfrak{A}|$ and each $R_{n}^{\mathfrak{B}}$ is $R_{n}^{\mathfrak{A}}$ restricted to $|\mathfrak{B}|$. The cardinal number of $\mathfrak{A}(\overline{\mathfrak{Q}})$ means that of $|\mathfrak{A}|$.

The symbols of the elementary language $L_{\rho}$ are the sentential connectives $\wedge, \vee, \sim, \rightarrow, \leftrightarrow$, the quantifiers $\forall, \exists$, the individual variables $v_{0}, v_{1}, \cdots$, the equality symbol $\approx$, and the $\rho_{n}$-ary relation symbols $R_{0}, R_{1}, \cdots$. A typical formula $\phi$ (of $L_{\rho}$ ), taking $\rho_{0}=2$, is

$$
\forall v_{1}\left(P_{0} v_{0} v_{1} \vee v_{0} \approx v_{1}\right) \text {, }
$$

which has $v_{0}$ as its only free variable. An example of a sentence $\sigma\left(\right.$ of $\left.L_{p}\right)$, i.e., of a formula with no free variables, is

$$
\exists v_{0} \forall v_{1}\left(P_{0} v_{0} v_{1} \vee v_{0} \approx v_{1}\right) \text {. }
$$

It is clear what we mean by saying that $\sigma$ is true in $\mathfrak{A}$, or $\mathfrak{A}$ is a model of $\sigma$; namely, in this case, that $\mathfrak{A}$ has a kind of first element. Similarly,

An address delivered before the Vancouver meeting of the Society on August 30, 1962, by invitation of the Committee to Select Hour Speakers for Far Western Sectional Meetings; received by the editors December 21, 1962. 
if $\phi$ has at most $v_{0}, \cdots, v_{n}$ as free variables, we may say that the elements $a_{0}, \cdots, a_{n}$ of $|\mathfrak{A}|$ satisfy $\phi$ in $\mathfrak{A}$, meaning in the above case that $a_{0}$ is a first element of $\mathfrak{A}$.

If $\mathscr{L}$ is any class of similar algebraic systems, the theory of $\mathscr{L}, T h \mathfrak{L}$, is the set of all sentences true in every member of $\&$. Dually if $\Sigma$ is any set of sentences, $\operatorname{Mod} \Sigma$ is the class of all models of every member of $\boldsymbol{\Sigma}$. A theory $T$ is called complete if $T=T h \mathfrak{A}$ for some single system A. Henceforth " $T$ " will always denote a complete theory, having infinite models.

The similar systems $\mathfrak{A}$ and $\mathfrak{B}$ are called elementarily indistinguishable $(\mathfrak{A} \equiv \mathfrak{B})$ if $T h \mathfrak{A}=T h \mathfrak{B}$. If $\mathfrak{B}$ is a subsystem of $\mathfrak{A}$, an even stronger relationship than $\mathfrak{Q} \equiv \mathfrak{B}$ sometimes holds, in which any elements $b_{0}, \cdots, b_{n-1}$ of $\mathfrak{B}$ satisfy an arbitrary formula in $\mathfrak{B}$ if and only if they satisfy it in $\mathfrak{A}$; we then say that $\mathfrak{B}$ is an elementary subsystem of $\mathfrak{A}(\mathfrak{B} \propto \mathfrak{A})$. Thus if $b$ is a first element of $\mathfrak{B}$ and $\mathfrak{B} \equiv \mathfrak{A}$ then $\mathfrak{A}$ has a first element, but $b$ itself need not be a first element of $\mathfrak{A}$, unless $\mathfrak{B} \propto \mathfrak{A}$.

With the help of these notions we now restate the Löwenheim. Skolem theorem in a stronger form due to A. Tarski, by whom the notions of elementary equivalence and elementary subsystems were first introduced : ${ }^{1}$

LöWENHEIM-SKolem THEOREM. If $X \subseteq|\mathfrak{A}|$ and $\bar{X} \leqq \kappa \leqq \overline{\mathfrak{A}}$ then there exists $\mathfrak{B} \propto \mathfrak{A}$ such that $X \subseteq|\mathfrak{B}|$ and $\overline{\mathfrak{B}}=\kappa$. Also, if $\kappa \geqq \overline{\mathfrak{A}}$ then there exists $\mathfrak{C} \times \mathfrak{A}$ such that $\mathfrak{A} \neq \neq \mathfrak{C}$ and $\mathbb{\mathfrak { C }}=\kappa .^{2}$

This theorem illustrates well the two directions in which the theory of models faces. It would obviously be classified as a theorem of general algebra, except for the fact that it involves some metamathematical notions. Actually, algebra has for a long time dealt with the metamathematical notions of polynomial and equation. The elementary language adds to these the sentential connectives and quantifiers, obtaining a much richer language, but still one so restricted that various strong general results hold concerning all properties expressible in it. On the other hand, the theory of models may be considered as a branch of the foundations of mathematics. If we take for $\mathfrak{A}$ in the Löwenheim-Skolem theorem the set of all sets or, more cautiously, the set of all sets of sets of sets of natural numbers, together with the $\epsilon$-relation, then we obtain the so-called Skolem paradox: $:^{1}$ there is a denumerable model for set theory, even though one of the valid sentences of set theory asserts the existence of a nondenumerable set.

\footnotetext{
${ }^{1}$ For references, see $[33 ; 34]$.

$2 \alpha$ and $\lambda$ always denote infinite cardinals.
} 
The Löwenheim-Skolem theorem happens also to be a typical result of the type I want to discuss today. Each of the results I shall discuss establishes some fact concerning the class of all models of an arbitrary complete theory $T$, usually, the existence of a model of $T$ of some special kind. Despite the fact that the class of models of a complete theory is, in one sense, a very general notion, with instances in every branch of algebra, the requirement of completeness is, in another sense, a very exacting one and, consequently, the classes $\operatorname{Mod} T$ have in common, as we shall see, a number of strong properties. ${ }^{3}$

Before turning to these general results, we should have before us some concrete examples of complete theories.

EXAMPLE 1. Let $\mathfrak{A}_{1}=\langle R t,\langle\rangle$ be the rational numbers with their usual ordering. By a result of Langford, ${ }^{1} \operatorname{Mod} T h \mathfrak{A}_{1}$ coincides with the class $\mathfrak{B}_{1}$ of all densely ordered systems with no first or last element. Viewed from another angle, Langford's result is that $T_{1}=T h \mathfrak{B}_{1}$ is complete.

ExAmple 2. Let $\mathfrak{A}_{2}=\langle C,+, \cdot\rangle$ be the field of complex numbers. Tarski [32] showed that $\operatorname{Mod} T h \mathfrak{A}_{2}$ is the class of all algebraically closed fields of characteristic zero.

EXAMPLE 3. Let $\mathfrak{A}_{3}=\langle R l,+, \cdot\rangle$ be the field of real numbers. Again by a result of Tarski [32], Mod Th $\mathfrak{A}_{3}$ is the class of all real closed fields.

EXAMPLE 4. Let $\mathfrak{H}_{4}=\langle R t,+, \cdot\rangle$ be the field of rationals. We know no exhaustive description of $\operatorname{Mod} T h \mathfrak{A}_{4}$ and, indeed, from a result of Julia Robinson, ${ }^{1}$ one can probably infer that we shall never have such a description (cf. [33, p. 716]).

The task of establishing that a given theory is complete is often a difficult one. The development of general metamathematical techniques for establishing completeness and their application in particular cases (where, in each case, a special mathematical or algebraic study must be made) form a major area of the theory of models. ${ }^{4} \mathrm{We}$ shall not discuss such problems here, except to mention one technique, which is not often applicable but, when it can be applied, is the easiest of all.

The theory $T_{1}=T h \mathfrak{A}_{1}$ of Example 1 is $\boldsymbol{\aleph}_{0}$-categorical, i.e., all models

3 For a summary of recent results in the theory of models which are concerned with the class of models of a (possibly incomplete) set $\boldsymbol{\Sigma}$ of sentences, and particularly with the form of the sentences in $\Sigma$, see [21]. We shall discuss here neither results lying in this area nor even certain results which fall both in this area and the area we are dealing with, such as the theorem of Robinson [27]. (For a discussion of interrelations between several theorems like Robinson's and the results in $\$ 1$ below, see [24].)

4 Cf., e.g., $[28 ; 32]$. 
of $T_{1}$ of power $\boldsymbol{\aleph}_{0}$ are isomorphic. This fact is the well-known theorem of Cantor concerning the order type $\eta$. (The type of argument Cantor used will be referred to as a Cantor-type argument.) $T_{1}$ is not $k$-categorical for any other $k$. On the other hand, $T_{2}=T h \mathfrak{A}_{2}$ is, by a theorem of Steinitz, $\kappa$-categorical for every $\kappa>\boldsymbol{\aleph}_{0}$, but not for $\kappa=\aleph_{0}$. The theory of algebraically closed fields of characteristic $p$ is $\kappa$-categorical for every $\kappa$. (These examples were mentioned in [19; $35]$.) As was remarked in $[19 ; 35]$, it follows easily from the Löwenheim-Skolem theorem that any theory having only infinite models, which is $\kappa$-categorical for some $\kappa$, must be complete.

Łoś [19] raised the question whether all theories $k$-categorical for some $k$ exhibit one of the three patterns above, that is:

\section{If $T$ is $\kappa$-categorical for some $\kappa>\aleph_{0}$, must $T$ be $\kappa$-categorical for all $\kappa>\boldsymbol{N}_{0}$ ?}

This problem turned out to be very difficult, and played a role as a sort of test problem which stimulated quite a bit of the work concerning models of arbitrary complete theories to be discussed in $\$ 1$, $\S 2$, and $\S 3$ below. In $\S 4$ we shall return to the problem itself.

There are three theorems which provide the basic tools for much of the theory of models. One is the Löwenheim-Skolem theorem. Most basic of all is the Compactness Theorem, a model-theoretic consequence of Gödel's completeness theorem. ${ }^{1}$ (For another proof of the Compactness Theorem, see $\S 6$.)

Compactness Theorem. If every finite subset of the set $\Sigma^{\prime}$ of sentences has a model, so has $\Sigma^{\prime}$.

If $a_{i}$, for $i \in I$, are members of $|\mathfrak{A}|$, we denote by $\left(\mathfrak{A}, a_{i}\right)_{i \in I}$ the system $\mathfrak{I}^{\prime}=\left\langle|\mathfrak{A}|, R_{0}^{\mathfrak{N}}, R_{1}^{\mathfrak{I}}, \cdots ; a_{i}\right\rangle_{i \in I}$ obtained by adjoining all the $a_{i}$ as distinguished elements. Although we are considering throughout theories $T$ with countably many symbols, an indispensable auxiliary role will be played by languages, like the language $L_{p, I}^{\prime}$ corresponding to $\mathfrak{X}^{\prime}$, having nondenumerably many symbols. By a result of Malcev, ${ }^{1}$ the Compactness Theorem extends to these languages.

The third basic theorem is due to Tarski [34]:

UnION TheOREM. The union

$$
U\{\mathfrak{X} / \mathfrak{A} \in Q\}=\left\langle U\{|\mathfrak{A}| / \mathfrak{A} \in Q\}, U\left\{R_{0}^{\mathfrak{A}} / \mathfrak{A} \in Q\right\}, \cdots\right\rangle
$$

of a $\gg$-directed family $Q$ is an elementary extension of each member of $Q$.

1. Homogeneous universal models. Suppose $\mathfrak{A}$ is a model of $T$ of power $\kappa . \mathfrak{U}$ is called universal if every model of $T$ of power $\leqq \kappa$ is 
isomorphic to an elementary submodel of $\mathfrak{A}$. $\mathfrak{A}$ is called homogeneous if, whenever $X \subseteq|\mathfrak{A}|, \bar{X}<\kappa$, and $(\mathfrak{A}, x)_{x \in X} \equiv(\mathfrak{A}, f(x))_{x \in X}$, then $f$ can be extended to an automorphism of $\mathfrak{A}$.

Theorem 1. (a) Any two homogeneous universal models of $T$ of the same power are isomorphic.

(b) (G.C.H.) $)^{5}$ There exists a homogeneous universal model of $T$ in each power $\boldsymbol{\aleph}_{\alpha+1}$.

Examples of homogeneous universal systems are the $\eta_{\alpha+1}$ ordered systems of Hausdorff and the $\eta_{\alpha+1}$ real closed fields of Erdös, Gillman, and Hendrickson [8]. (In both of these cases, there is also homogeneity (and universality) in a purely algebraic sense, as the theories $T_{1}$ and $T_{3}$ are model-complete, i.e., $\mathfrak{A}, \mathfrak{B} \in \operatorname{Mod} T$ and $\mathfrak{A} \subseteq \mathfrak{B}$ implies $\left.\mathfrak{A} \propto \mathfrak{B} .{ }^{6}\right)$

Theorem 1 is due to B. Jónsson $[15 ; 16]$, and M. Morley and myself $[24]$. In $[15 ; 16]$, a general, purely algebraic theorem is proved to the effect that a class $\&$ of relational systems which obeys certain postulates has one and, up to isomorphism, only one, "\&-homogeneous L-universal" member of power $\boldsymbol{\aleph}_{\alpha+1}$. In [24] it is shown that for any $T$, the class Mod $T$ or, rather, a certain variant of it (cf. [21]) always fulfills Jónsson's postulates. In these postulates the notion "elementary subsystem" does not, of course, occur, but rather the notion "subsystem belonging to 2. ." Roughly speaking the postulates are (i) an analogue of the Löwenheim-Skolem theorem, (ii) an analogue of the Union Theorem, and (iii) an analogue of certain consequences of the Compactness Theorem. Postulate (iii) states, in fact, that any two members of $\&$ can be isomorphically imbedded in a third and in such a way as to preserve the elements of a given common $\mathcal{L}$-subalgebra (embedding with amalgam). By a well known theorem of Schreier, the class of all groups fulfills these postulates (cf. [15]). It is interesting that some of the important properties of the class of all groups and some of the important properties of arbitrary classes $\operatorname{Mod} T$ can be derived from the same postulates, so that such a result in either of the two fields can be translated into a result in the other.

Theorem $1 \mathrm{~b}$ is also valid for inaccessible powers $>\boldsymbol{N}_{0}$. In accessible powers $\aleph_{\lambda}, \lambda$ a nonzero limit ordinal, a unique isomorphism

\footnotetext{
- This notation indicates that (b) is proved using the generalized continuum hypothesis.

- The notion of model-completeness was used by A. Robinson [28] to give a shorter proof of the completeness of $T_{3}$ than Tarski's [32]. S. Kochen [18] found a still shorter proof involving the $\eta_{\alpha}$-real closed fields.
} 
type can (assuming G.C.H.) still be singled out of $\operatorname{Mod} T$ (cf. [24]), though its members need not be homogeneous. Before discussing the case $\boldsymbol{\aleph}_{0}$ it is necessary to define some notions, which will also be involved in $\$ 2$ and $\$ 3$.

Given an element $a$ of $|\mathfrak{A}|$, it is clearly appropriate to call the set $Q_{\mathfrak{A}, a}=\{\phi / a$ satisfies $\phi$ in $\mathfrak{A}\}$ the (elementary) type of the element $a$ (in $\mathfrak{2})$. The set of formulas $\phi$ with at most $v_{0}$ free forms a Boolean algebra $B_{0}$ under disjunction, conjunction, and negation, $\theta$ and $\phi$ being identified if $\forall v_{0}(\theta \leftrightarrow \phi) \in T=T h \mathfrak{A}$. $Q_{\mathfrak{A}, a}$ is a dual prime ideal in $B_{0}$ and moreover, by the Compactness Theorem, every dual prime ideal of $B_{0}$ is of this form. Thus the set of types of elements forms the Stone space, $S t_{0} T$, of $B_{0},{ }^{7}$ i.e., the compact zero-dimensional Hausdorff space associated with $B_{0}$ by Stone's well-known construction. If $P \in S t_{0} T$, let $P(\mathfrak{A})=\left\{a / P=Q_{\mathfrak{A}, a}\right\} . P(\mathfrak{H})$ may be empty for some models $\mathfrak{A}$ of $T$. For example, in a real-closed field all the "nonArchimedean" elements $x$, such that $x>1, x>1+1, \cdots$, form a single type $P$ of element [32], but the real field $\mathfrak{A}_{3}$ omits $P$, i.e., $P\left(\mathfrak{H}_{3}\right)=0$.

$S t_{n} T(n=1,2, \cdots)$ is similarly defined as the space of types of $n$-tuples of elements. The disjoint union $S t_{0} T \cup S t_{1} T \cup \ldots$ is called StT.

THEOREM $2[36 ; 24]$. T has a denumerable homogeneous universal model if and only if StT is countable.

For homogeneous universal models there is an alternative characterization, due in part to Keisler. Let us call a model $\mathfrak{A}$ of $T$ saturated if whenever $X \subseteq|\mathfrak{X}|$ and $\bar{X}<\kappa$, the system $\mathfrak{U}^{\prime}=(\mathfrak{A}, x)_{x \in X}$ has all possible types of elements, i.e., $P\left(\mathfrak{H}^{\prime}\right) \neq 0$ for every $P \in S t_{0}$ Th $\mathfrak{X}^{\prime}$.

Theorem $3[17 ; 24 ; 36]$. An infinite model $\mathfrak{A}$ of $T$ is homogeneous universal if and only if $\mathfrak{A}$ is saturated.

The results to which we now turn lie in just the opposite direction from saturation; they assert that models exist which omit a certain type or types of elements.

\section{Omitting types of elements.}

Theorem 4. If $P \in S t_{0} T$ is not an isolated point, then $T$ has a denumerable model $\mathfrak{Q}$ such that $P(\mathfrak{U})=0$. Indeed, if $W$ is any set of the first category in StT, then, for some denumerable model $\mathfrak{A}$ of $T, P(\mathfrak{A})=0$ for all $P \in W$.

${ }^{7}$ This construction of $B_{0}$ and $S t(T)$ is a special case of a construction outlined in [33]. 
Theorem 4 originated in some work of Ryll-Nardzewski (see Theorem 5 below). It was first stated explicitly, in one form, and proved by Ehrenfeucht (cf. [25]), and later, independently by Engeler [5], and Svenonius [30]. A more general form was found by myself [36], and the most general form (of which 4 is a version) was found by Svenonius [31] and Engeler [6;7].

Theorem 4 can be proved by a modification of Henkin's proof of the Compactness (or Completeness) Theorem [14]. To the set $T$ of sentences we add the sentences $\exists v_{0} \phi_{n}\left(v_{0}\right) \rightarrow \phi_{n}\left(c_{n}\right)$, just as in Henkin's proof. At the same time, however, we add consistently additional conditions $\theta\left(c_{n}\right)$ at each step. Henkin showed that the new set of sentences has a model in which every element is denoted by some $c_{n}$. Thus, by adding the $\theta\left(c_{n}\right)$ we can try to ensure that every $a \in|\mathfrak{A}|$ has a certain property (e.g., $a \notin P(\mathfrak{U})$ ). It seems to me likely that this general method of proof is one which has not been fully exploited.

Theorem 4 has a number of consequences. One was the original result of Ryll-Nardzewski [29] (cf. also [5; 30]):

Theorem 5. $T$ is $\aleph_{0}$-categorical if and only if for each $n, S t_{n} T$ is finite.

It is the "only if" that follows easily from Theorem 4; the "if" is proved by using a Cantor-type argument.

Let us call a model $\mathfrak{U}$ of $T$ prime if every model of $T$ has an elementary subsystem isomorphic to $\mathfrak{A}$. Using Theorem 4 twice, together with some more Cantor-type arguments, one can prove:

Theorem $6[30 ; 31 ; 36]$. (a) Any two prime models of $T$ are isomorphic, and each prime model is homogeneous.

(b) $\mathfrak{A}$ is prime if and only if, for any $a_{0}, \cdots, a_{n} \in|\mathfrak{A}|, Q_{\mathfrak{A}, a_{0}, \ldots, a_{n}}$ is an isolated point.

(c) $T$ has a prime model if and only if the isolated points are dense in $S t T$.

For some further consequences of Theorem 4, and a discussion of Theorem 4 from the point of view of languages with infinite conjunctions, ${ }^{8}$ see $[6 ; 7]$.

3. Applications of Ramsey's theorem. Ehrenfeucht and Mostowski [4] made the first application to general model theory of the wellknown theorem of Ramsey: if the set $A^{(n)}$ of all n-element subsets of an infinite set $A$ is partitioned into finitely many parts $Y_{1}, \cdots, Y_{k}$,

8 In the last few years a number of significant results have been obtained concerning the theory of models for languages richer in one way or another than the elementary language. We shall consider throughout only the elementary language. 
then some $Y_{j}$ includes the whole $B^{(n)}$ for some infinite $B \subseteq A$. By applying Ramsey's theorem together with the Compactness Theorem, while using the so-called Skolem functions, they proved the existence of models of $T$ with "many" automorphisms:

THEOREM 7. Given any ordered system $\langle X,<\rangle$ of power $\kappa$, there is a model $\mathfrak{A}$ of $T$ of power $\kappa$ such that $X \subseteq|\mathfrak{A}|$ and every automorphism of $\langle X,<\rangle$ can be extended to an automorphism of $\mathfrak{A}$.

Two elements $a$ and $b$ of a system $\mathfrak{A}$ might be said to have the same absolute (as opposed to elementary) type (in $\mathfrak{l}$ ) if for some automorphism $f$ of $\mathfrak{A}, f(a)=f(b)$. Using Theorem 7 (and its proof) Ehrenfeucht [3] obtained the following result:

THEOREM 8. $T$ has in each power $\kappa\left(>\aleph_{0}\right)$ a model $\mathfrak{A}$ such that for any finite $X \subseteq|\mathfrak{A}|$, the system $(\mathfrak{A}, x)_{x \in X}$ has only countably many $a b$ solute types of elements.

A fortiori, ( $(, x)_{x \in X}$ has countably many (elementary) types of elements. Morley $[\mathbf{2 3}]^{9}$ has obtained a strengthening of this consequence of Theorem 8, namely:

THEOREM 9. Thas in each power $\kappa$ a model $\mathfrak{A}$ such that for any countable $X \subseteq|\mathfrak{A}|,(\mathfrak{A}, x)_{x \in X}$ has only countably many types of elements.

It is not known whether "finite" can be replaced by "countable" in Theorem 8 itself.

There are a number of natural conjectures which would strengthen Theorem 4 or 6 . For several of these, G. Fuhrken has constructed ingenious counterexamples. In [10] he showed that Theorem 4 fails altogether for languages with uncountably many symbols. In [11; 12], he showed that the system $\mathfrak{A}$ of Theorem 4 cannot always be obtained as an elementary submodel of an arbitrary given model; and that a model of $T$ may be minimal (have no proper elementary submodels) but not prime.

The situation regarding an "upward" version of Theorem 4 is not yet clear. The real number field $\mathfrak{H}_{3}$ and the type $P$ of non-Archimidean elements show that $T$ may have a model $\mathfrak{A}$ in power $2^{N_{0}}$ but no higher power with $P(\mathfrak{C})=0$. It seems likely, but is not yet established, that the same thing can happen for cardinals $>2^{N_{0}}$. (Morley has constructed various examples of theories $T$ having a model $\mathscr{A}$ of a certain power $\kappa>2^{N_{0}}$ but no higher power in which $P(\mathfrak{U})=0$ for all members $P$ of some set $W$.) On the other hand Theorem 8 is itself,

- A short summary of Morley's thesis [23] will appear soon in Proc. Nat. Acad. Sci., and the full thesis will be published in the near future. 
in a sense, an upward form of Theorem 4. Many theories $T$ have a continuum of types of elements. From Theorem 8 it easily follows that there is a countable subset $W$ of $S t_{0} T$ such that in each power there is a model of $T$ having exactly the types of elements in $W$. Can $W$ be described or characterized more explicitly?

Still another result whose proof involves Ramsey's Theorem is the following theorem about theories categorical in power.

THeOREM 10. If $T$ is $\kappa$-categorical for some $\kappa>\boldsymbol{\aleph}_{0}, \mathfrak{A}=\langle A, R, \cdots\rangle$ is a model of $T$, and $B$ is an infinite subset of $A$ then $R$ cannot be an ordering of $B$; more generally, if $R$ is n-ary it cannot be that, for any distinct $b_{1}, \cdots, b_{n} \in B, R$ holds for some permutation of the $b_{i}$ 's and fails for another.

Theorem 10 was proved by Ehrenfeucht [2] for $\kappa=2^{\aleph \alpha} ;$ D. Scott shows that $k=\kappa^{N 0}$ suffices; finally, Morley [23] showed that it is valid for any $\kappa>\boldsymbol{\aleph}_{0}$.

4. Theories categorical in power $\kappa>\boldsymbol{\aleph}_{0}$. By combining the results of $\S \S 1,2,3$, one can easily derive the two following theorems.

THEOREM 11. If $T$ has fewer than $2^{\text {No }}$ non-isomorphic models in some power $\kappa$ (in particular, if $T$ is $\kappa$-categorical), then StT is countable [3]; hence $T$ has a prime model $[30 ; 36]$ and a denumerable saturated model [36].

THEOREM 12. T cannot have exactly two non-isomorphic denumerable models [36].

Examples due to Ehrenfeucht (cf. [36]) show that Theorem 12 holds only for $n=2$.

Although a number of the results which have now been mentioned provide some information about a theory $T$ categorical in some power $\kappa>\mathfrak{Y}_{0}$, they are a long way from resolving Łoś' conjecture mentioned earlier that such a theory must be categorical in every nondenumerable power. This difficult problem was finally solved positively in the past year by Michael Morley, in his dissertation $[22 ; 23] .{ }^{9}$ In fact, Morley established (without the G.C.H.) the stronger result (cf. Theorems 1 and 3):

THEOREM 13. If $T$ is $\kappa$-categorical for some $\kappa>\aleph_{0}$, then every nondenumerable model of $T$ is saturated.

The beautiful proof of this result obtained by Morley is too long to be even roughly described here. (The improvements due to Morley, incorporated in Theorems 9 and 10 above, form a small but needed 
part of the proof.) However, I shall say a few words about what is probably the single most essential feature of the proof. Suppose $\mathfrak{U} \in \operatorname{Mod} T, X_{1} \subseteq X_{2} \subseteq|\mathfrak{A}|, T_{1}=\operatorname{Th}(\mathfrak{A}, x)_{x \in X_{1}}$ and $T_{2}=\operatorname{Th}(\mathfrak{A}, x)_{x \in X_{2}}$. Then, as Morley observed, there is a natural continuous map of $S t_{0} T_{2}$ onto $S t_{0} T_{1}$. Instead of considering only $S t T$, Morley associates with $T$ the category formed by all such maps. He applies simultaneously to all the spaces $S t_{0} T_{1}$ a construction analogous to the formation of the usual transfinite sequence of derivatives or derived sets of a topological space. To obtain the $\xi+1$ st "derivative" of one space in the category, one must know already what the $\xi$ th "derivative" is for every space in the category. For this new notion of "derivative," Morley establishes an analogue of the Cantor-Bendixson Theorem. It follows, using also Theorem 9 above, that if $T$ is $\kappa$-categorical for some $\kappa>\aleph_{0}$, then there is an ordinal $\xi<\omega_{1}$, such that the $\xi$ th "derivative" of every space in the category vanishes. (A theory $T$ with the latter property is called totally transcendental, by an analogy with the special case in Example 2 above.) The rest of the proof makes use of the consequent fact that, for each $T_{1}$ (as above), every $P \in S t_{0} T_{1}$ has an "order $\eta$ of transcendence," i.e., $P$ is first removed in forming the $\eta+1$ st derivative.

Though "totally transcendental" is a very strong property, it does not imply that $T$ is categorical in nondenumerable powers. Indeed, from Morley's proof there does not appear to emerge any nice answer to the problem raised by Mostowski [25] of characterizing theories categorical in nondenumerable powers (cf. Theorem 5). While this question might have no nice answer, there are also still a number of unanswered yes-or-no questions concerning categorical theories, which have been raised by various people. For example: Suppose $T$ is $\boldsymbol{\aleph}_{1}$-categorical but not $\boldsymbol{\aleph}_{0}$-categorical.

Must $T$ have exactly $\aleph_{0}$ nonisomorphic denumerable models?

Can $T$ be finitely axiomatizable?

5. Löwenheim-Skolem theorems for pairs of cardinals. In this section we consider theories $T$ such that for each model $\mathfrak{A}$ of $T, R_{0}^{\mathfrak{P}}$ is an infinite subset of $|\mathfrak{A}|$. (It would be no more general to assume $U$ is the subset of $|\mathfrak{A}|$ defined by some fixed formula.) It is an easy consequence of the Compactness Theorem that $T$ has a model $\mathfrak{A}$ in each power $\kappa$ with $\bar{R}_{0}^{\mathfrak{A}}=\overline{\mathfrak{A}}$. It is also simple to construct a theory in all of whose models $\mathfrak{A}, \bar{R}_{0}^{\mathfrak{A}}=\overline{\mathfrak{A}}$. If $T$ has'a model $\mathfrak{A}$ of power $\kappa$ such that $\bar{R}_{0}^{\mathfrak{A}}=\lambda<\kappa$, we shall say $T$ admits $\kappa, \lambda$. I showed:

Theorem 14. If $T$ admits $\kappa, \lambda$ then $T$ admits $\aleph_{1}, \aleph_{0}$.

The proof of Theorem 14 (cf. [24]) uses a generalization (due partly 
to W. Craig) of Theorem 1, which concerns models which are homogeneous but not universal.

Theorem 14 leads to the general question: If $T$ admits $\kappa, \lambda$, what other pairs $\kappa^{\prime}, \lambda^{\prime}$ must $T$ admit? From the Löwenheim-Skolem theorem, it follows at once that if $T$ admits $\kappa, \lambda$ and $\kappa>\kappa^{\prime}>\lambda$ then $T$ admits $\kappa^{\prime}, \lambda$. R. Robinson constructed examples (cf. [24]) of theories $T_{1}, T_{2}, \cdots$ such that $T_{1}$ admits exactly those pairs $\kappa, \lambda$ with $\kappa \leqq 2^{\lambda}$, $T_{2}$ those with $\kappa \leqq 2^{2^{\lambda}}$, etc. Assuming the G.C.H., these are the only negative results known. (Morley has observed that, without assuming the G.C.H., a theory $T$ can be found which admits all and only the pairs $\boldsymbol{\aleph}_{\alpha+1}, \boldsymbol{\aleph}_{\alpha}$, and that various other examples result from finite iteration of his and Robinson's constructions.) By using a method completely different from the proof of 14, Chang and Keisler [1] have obtained the only other positive results known. Assuming the G.C.H., their results may be summarized as follows:

Theorem 15. (G.C.H.) Suppose T admits $\kappa, \lambda$. Then:

(a) If $\kappa>\lambda^{\prime}>\lambda$, then T admits $\kappa, \lambda^{\prime}$.

(b) If cf $\kappa<c f \lambda$, then $T$ admits $2^{\kappa}, \lambda$.

(c) If cf $\kappa=c f \lambda$, then $T$ admits $2^{\kappa}, 2^{\lambda} .{ }^{10}$

The proof of Chang and Keisler relies on a construction called the ultraproduct, which will be discussed in the final section, $\S 6$.

G. Fuhrken $[11 ; 12 ; 13]$ has shown that the results or methods of Theorems 14 and 15 can be used to obtain strong conclusions (one of which generalizes 14) concerning languages which involve an additional quantifier "there exist at least $\boldsymbol{\aleph}_{\alpha} x$ such that."

As indicated above, it is an open problem whether there are other theorems of the form of 14 and 15. For example: if $T$ admits $\boldsymbol{\aleph}_{1}, \boldsymbol{\aleph}_{0}$, must $T$ admit $\boldsymbol{\aleph}_{\alpha+1}, \boldsymbol{\aleph}_{\alpha}$ for every (some) $\alpha>0$ ? In another direction, Chang raised the interesting question whether 14 can be improved (in analogy to the Löwenheim-Skolem theorem) as follows: does every model $\mathfrak{A}$ of $T$ with $\bar{R}_{0}^{\mathfrak{A}} \neq \overline{\mathfrak{A}}$ have an elementary submodel $\mathfrak{B}$ with $\overline{\mathfrak{B}}=\boldsymbol{\aleph}_{1}$ and $\bar{R}_{0}^{\Re}=\boldsymbol{\aleph}_{0}$ ? By using Skolem functions one easily sees that this problem is really purely algebraic or set-theoretical. A negative answer to the problem is an easy consequence of a positive answer to the following question: Does there exist a family $W$ of subsets of a set $A$ of power $\boldsymbol{\aleph}_{1}$ such that $\bar{W}=\boldsymbol{\aleph}_{2}$ while, for any countable $B \subseteq A,\{X \cap B / X \in W\}$ is countable? The latter question is a version of a problem raised by Kurepa in 1943 and still unresolved (cf., e.g., $[26$, p. 344]).

${ }^{10} \mathrm{cf} . \kappa$ (the character of confinality of $\kappa$ ) is the smallest cardinal $\mu$ such that $\kappa$ can be expressed as $\sum\left(\lambda_{j} / j_{\epsilon} J\right)$, where $\bar{J}, \lambda_{j}<\kappa$ for each $j_{\epsilon} J$. 
6. Ultraproducts. The study of ultraproducts is an important chapter of the theory of models, commenced by Łos [20]. It is impossible to do here any justice to this extensive topic. (A detailed study of the basic properties of ultraproducts can be found in [9].) However, in this concluding section, I shall at least say what ultraproducts are, and will mention three results concerning them, which are directly related to the topics of $\S \S 5$ and 1.

Suppose that $\mathfrak{A}^{j}(j \in J)$ is an indexed family of similar relational systems, and that $F$ is an ultrafilter for the set $J$. The ultraproduct $\prod_{F}\left(\mathfrak{A}^{j} / j \in J\right)$ is the system ( $\mathbb{f}$ formed as follows: If $f$ and $g$ are members of the Cartesian product $C=\prod\left(\left|\mathfrak{A}^{j}\right| / j \in J\right)$, put $f \equiv_{F} g$ if $f(j)=g(j)$ for $F$-almost all $j \in J$, i.e., if $\{j / f(j)=g(j)\} \in F$. $|\mathbb{E}|$ is the set of equivalence classes $f^{\prime}=f / \equiv_{F}$ of members $f$ of $C$. Similarly, if the $n$th relations of the $\mathfrak{A}^{i}$ are, say, ternary, we can let $R_{n}^{\mathbb{G}} f^{\prime} g^{\prime} h^{\prime}$ if and only if $R^{\mathfrak{2} j} f(j) g(j) h(j)$ for $F$-almost all $j \in J$.

The definition of the ultraproduct makes no reference to metamathematical notions. Nevertheless, it turns out that the relationship involved in the definition extends to all elementary formulas. Theorem 16 , below, expresses this fact for sentences.

TheOREM 16 [20]. $\sigma$ is true in $\prod_{F}\left(\mathfrak{H}^{i} / j \in J\right)$ if and only if $\sigma$ is true in $\mathfrak{A}^{j}$ for $F$-almost all $j \in J$.

The corresponding situation for formulas can be reduced to that for sentences, by means of the following easily verified fact: If $f \in C$, then $\prod_{F}\left(\left(\mathfrak{H}^{i}, f(j)\right) / j \in J\right) \cong\left(\mathfrak{C}, f^{\prime}\right)$. From this and Theorem 16 it follows that, for any system $\mathfrak{A}$, each ultrapower $\mathfrak{A}_{F}^{J}=\prod_{F}(\mathfrak{A} / j \in J)$ is isomorphic to an elementary extension of $\mathfrak{A}$.

One can derive the Compactness Theorem from Theorem 16 (cf. [9]).

The proof of each of the basic existence theorems discussed in the preceding sections involves, of course, a method for constructing models. The ultraproduct is another such method, but one distinguished by its generality and simplicity and, especially, by the fact that it is a purely algebraic construction. Moreover, the ultraproduct yields a new model which is closely related to the given (factor) models. In particular, it is easily seen that, if $R_{0}^{\mathscr{R}}=U \subseteq|\mathfrak{A}|, \mathfrak{E}=\mathfrak{R}_{F}^{J}$, and $X=U_{F}^{J}$, then $\bar{X}=\overline{R_{0}^{c}}$. From this fact together with the following theorem, Chang and Keisler deduced Theorem 15 of $\$ 5$. (For other results and problems concerning the cardinalities of ultraproducts, see [9].)

Theorem 17 [1]. If $\bar{J}=\lambda$, there is an ultrafilter $F$ for $J$ such that, for any $\mathfrak{A}$ of arbitrary power $\kappa, \mathfrak{Y}_{F}^{J}$ has power $\kappa^{\lambda}$. 
Perhaps the deepest result which has been obtained concerning ultraproducts is the following theorem of Keisler [17]:

THEOREM 18 (G.C.H.). If $\bar{J}=\boldsymbol{\aleph}_{\alpha}$ then there is an ultrafilter $F$ for $J$ such that, for any infinite systems $\mathfrak{A}_{j}(j \in J)$, each of power $\leqq \boldsymbol{\aleph}_{\alpha+1}$, the ultraproduct $\prod_{F}\left(\mathfrak{A}^{i} / j \in J\right)$ is a saturated system of power $\boldsymbol{\aleph}_{\alpha+1}$.

An immediate consequence of Theorem 18 is the following corollary (for which Keisler [17] also gave a direct proof).

Corollary 19 (G.C.H.). $\mathfrak{A} \equiv \mathfrak{B}$ (if and) only if $\mathfrak{A}$ and $\mathfrak{B}$ have isomorphic ultrapowers.

Corollary 19 provides a solution for an old problem of Tarski's, that of finding a purely algebraic condition which is equivalent to the basic relationship $\mathfrak{A} \equiv \mathfrak{B}$ of the theory of models.

For ultraproducts of the power of the continuum, a much stronger result than Theorem 18 holds. Indeed, Keisler has shown (cf. [17], and also the discussion in [9] of countably complete ultrafilters):

TheOREM 20 (G.C.H.). If the ultraproduct $\mathfrak{E}=\prod_{F}\left(\mathfrak{A}_{j} / j \in J\right)$ is of the power $\mathfrak{\aleph}_{1}$, then either $\mathfrak{S}$ is isomorphic to one of the factors $\mathfrak{A}_{j}$ or $\mathfrak{C}$ is saturated.

From another point of view, Theorem 20 is dramatic evidence of the limitations of the ultraproduct. It clearly implies that, for many purposes, other methods of constructing models are essential.

Added in proof, March 26, 1963. The problem raised just after Theorem 9 has been answered affirmatively by J. Silver. Two more theorems of the form of Theorems 14 and 15 have been established, answering some of the questions raised in $\$ 5: \mathrm{C}$. C. Chang has shown that (G.C.H.) if $T$ admits $\boldsymbol{N}_{1}, \boldsymbol{N}_{0}$ then $T$ admits $\boldsymbol{N}_{\alpha+1}, \boldsymbol{N}_{\alpha}$ for every regular $\boldsymbol{N}_{\alpha}$. Let $2_{0}=\lambda$ and $2_{n+1}=2^{2 n}$. The author has shown that if, for every $n, T$ admits a pair $\kappa, \lambda$ such that $k \geqq 2_{n}$, then $T$ admits every pair $\kappa, \lambda(\kappa>\lambda)$.

\section{BibLIOGRAPHY}

1. C. C. Chang and H. J. Keisler, Applications of ultraproducts of pairs of cardinals to the theory of models, Pacific J. Math. 12 (1962), 835-845. 248.

2. A. Ehrenfeucht, On theories categorical in power, Fund. Math. 44 (1957) 241-

3. - Theories having at least continuum many nonisomorphic models in each infinite power, Notices Amer. Math. Soc. 5 (1958), 680-681.

4. A. Ehrenfeucht and A. Mostowski, Models of axiomatic theories admitting automorphisms, Fund. Math. 43 (1956), 50-68.

5. E. Engeler. Äquivalenzklassen von $n$-Tuplen, Z. Math. Logik Grundlagen Math. 5 (1959), 340-345. 
6. - Unendliche Formeln in der Modeltheorie, Z. Math. Logik Grundlagen Math. 7 (1961), 154-160.

7. - Ein Reduktionstheorem filr unendliche Formeln, Math. Ann. (to appear).

8. P. Erdös, L. Gillman, and M. Hendrickson, An isomorphism theorem for real closed fields, Ann. of Math. 61 (1955), 542-547.

9. T. Frayne, A. Morel, and D. Scott, Reduced direct products, Fund. Math. (to appear).

10. G. Fuhrken, Bemerkung zu einer Arbeit E. Engelers, Z. Math. Logik Grundlagen Math. 8 (1962), 91-93.

11. - First-order languages with a generalized quantifier. Minimal models of first-order theories, Doctoral dissertation, University of California, Berkeley, Calif., 1962.

12. - On minimal models of complete theories, Notices Amer. Math. Soc. 9 (1962), 146. On generalized quantifiers, Ibid. p. 132. A Skolem-type normal form for languages with a generalized quantifier, Ibid., pp. 320-321.

13. G. Fuhrken and R. Vaught, Non-characterizability of the ordering of the natural numbers, Notices Amer. Math. Soc. 9 (1962), 321.

14. L. Henkin, The completeness of the first order functional calculus, J. Symb. Logic 14 (1949), 159-166.

15. B. J6nsson, Universal relational systems, Math. Scand. 4 (1956), 193-208.

16. - Homogeneous universal relational systems, Math. Scand. 8 (1960), 137-142.

17. H. J. Keisler, Ultraproducts and elementary classes, Indag. Math. 23 (1961), 477-495.

18. S. Kochen, Ultraproducts in the theory of models, Ann. of Math. 74 (1961), 221261.

19. J. Los, On the categoricity in power of elementary deductive systems and some related problems, Colloq. Math. 3 (1955), 58-62.

20. - Quelques remarques, thêorèmes, et problèmes sur les classes dêfinissables d'algèbres, Mathematical Interpretation of Formal Systems, pp. 98-113, North Holland Publ. Co., Amsterdam, 1955.

21. R. Lyndon, Properties preserved under algebraic constructions, Bull. Amer. Math. Soc. 65 (1959), 287-299.

22. M. Morley, Categoricity in power, Notices Amer. Math. Soc. 9 (1962) 218.

23. - Categoricity in power, Doctoral dissertation, University of Chicago, Chicago, Ill., 1962.

24. M. Morley and R. Vaught, Homogeneous universal models, Math. Scand. 11 (1962) (to appear).

25. A. Mostowski, Quelques observations sur l'usage des methodes non finitistes dans la méta-mathématique, Colloq. Internat. du C. N. R. S., 1955, vol. 70, Paris, 1958, pp. 19-32.

26. R. Ricabarra, Conjuntos ordenados y ramificados, Bahla Blanca, Instituto de Mathematica, Universidad Nacional del Sur, 1958.

27. A. Robinson, $A$ result on consistency and its application to the theory of definition, Indag. Math. 18 (1956), 47-58.

28. - Complete theories, North Holland Publ. Co., Amsterdam, 1956.

29. C. Ryll-Nardzewski, On theories categorical in power $\leqq \aleph_{0}$, Bull. Acad. Polon. Sci., Sér. Math. Astr. Phys. 7 (1959), 545-548.

30. L. Svenonius, No-categoricity in first order predicate calculus, Theoria 25 (1959), 82-94. 
31. - On minimal models of first order systems, Theoria 26 (1960), 44-52.

32. A. Tarski, $A$ decision method for elementary algebra and geometry, 2nd ed., Univ. of Calif. Press, Berkeley, Calif., 1951.

33. - Some notions and methods on the borderline of algebra and metamathematics, Proc. Internat. Congr. Math. 1950, 1 (1950), 705-720.

34. A. Tarski and R. Vaught, Arithmetical extensions of relational systems, Compositio. Math. 13 (1957), 81-102.

35. R. Vaught, Applications of the Löwenheim-Skolem theorem to problems of completeness and decidability, Indag. Math. 16 (1954), 467-471.

36. - Denumerable models of complete theories, Proc. Sympos. on Foundations of Mathematics: Infinitistic Methods, held in Warsaw, 1959, Pergman Press, Krakow, 1961, pp. 303-321.

University of California, Berkeley 